\title{
FIXED POINTS AND STATIONARY POINTS OF DISSIPATIVE MULTIVALUED MAPS
}

\author{
JEAN-PIERRE AUBIN AND JERROLD SIEGEL
}

\begin{abstract}
Fixed or stationary points of multivalued maps are seen to exist in the presence of various entropy functions for the map. Generalizations of the BanachPicard and Caristi fixed point theorems are presented.
\end{abstract}

1. Introduction. Let $E$ be a complete metric space and let $F(E)$ denote the set of nonempty subsets of $E$. Let $A: E \rightarrow F(E)$ be a given multivalued map on $E$. Regarding $E$ as a set of "states", we may consider the discrete dynamical system defined by $A$, which associates to any state $x$ the set of states $A(x)$ which can be reached in one time period. Examples of such systems are supplied by control theory where, given a map $f: E \times U \rightarrow E$ where $U$ is a set of "controls", one sets $A(x)=\{f(x, u) \mid u \in U\}$.

We will address ourselves to the problem of existence of fixed points $\bar{x} \in A(\bar{x})$ (which may be interpreted as rest-points of the system), and the existence of stationary points $\left\{x_{*}\right\}=A\left(x_{*}\right)$ (end-points of the system). In the framework of control theory, $\bar{x}$ is a fixed point if $\exists \bar{u} \in U$ such that $\bar{x}=f(\bar{x}, \bar{u})$, and $x_{*}$ is a stationary point if $\forall u \in U, x_{*}=f\left(x_{*}, u\right)$.

We present only basic results which have intrinsic interest. Moreover, these results may be categorized in terms of which of the various concepts of "entropy" (or "strong Liapunov functions") we assume for $A$.

These kinds of results may be useful for computational methods of solutions to optimization problems (see E. Polac [7] and W. Zangwill [11] for instance) and in system theory (see J. Willems [10] for instance).

2. Weakly dissipative systems. In this paper, we assume once and for all that $E$ is a complete metric space. We begin by defining what is meant by a weak dissipative system.

Definition 2.1. A function $\psi: E \rightarrow R_{+}$is called a weak entropy of $A$ if

$$
\forall x \in E, \exists y \in A(x) \text { such that } d(x, y) \leqslant \psi(x)-\psi(y) .
$$

A map $A: E \rightarrow F(E)$ is said to be weakly dissipative if there exists a weak entropy of $A$.

Note that we use the concept of entropy with a sign opposite to the one used by physicists. We also need the following.

Received by the editors December 6, 1978.

AMS (MOS) subject classifications (1970). Primary 47H10; Secondary 54C60.

Key words and phrases. Multivalued maps, contraction theorem, entropy, complete metric space. 
Definition 2.3. A sequence $\left\{x_{n}\right\}_{n \geqslant 0}$ of the elements in $E$ is called a trajectory starting at $x$ if $x_{0}=x$ and $x_{n+1} \in A\left(x_{n}\right), n \geqslant 0$. We denote the set of all such trajectories by $\mathcal{T}(A, x)$.

The following theorem is a typical application of "entropy".

THEOREM 2.4. Any weakly dissipative closed map $A: E \rightarrow F(E)$ has a fixed point; furthermore, for any $x_{0} \in E$, there exists a trajectory starting at $x_{0}$ and converging to a fixed point.

Proof. Let $\psi$ be a weak entropy of $A$. Given any $x \in E$ we may use (2.2) to construct $\left\{x_{m}\right\} \in \mathcal{T}(A, x)$ such that

$$
d\left(x_{p}, x_{q}\right) \leqslant \sum_{n=p}^{q-1} d\left(x_{n}, x_{n+1}\right) \leqslant \psi\left(x_{p}\right)-\psi\left(x_{q}\right) .
$$

Since the sequence $\psi\left(x_{n}\right)$ is nonincreasing and bounded from below $\left\{x_{n}\right\}$ is a Cauchy sequence.

Let $\bar{x}=\lim x_{n}$. Since $x_{n+1} \in A\left(x_{n}\right)$ we have that $(\bar{x}, \bar{x})$ is in the closure of the graph of $A$ and hence, by hypothesis, in the graph itself. Therefore $\bar{x} \in A(\bar{x})$.

We are also able to give a partial characterization of those $A$ which admit a weak entropy, hence are weakly dissipative. The characterization is in terms of the following function $\psi_{0}: E \rightarrow[0,+\infty]$ :

$$
\psi_{0}(x)=\inf \left\{\sum_{n=0}^{\infty} d\left(x_{n}, x_{n+1}\right) \mid\left\{x_{n}\right\} \in \mathcal{T}(A, x)\right\} .
$$

By a simple argument similar to that of Theorem 2.4 we have:

Proposition 2.6. If $A$ is weakly dissipative and if $\psi$ is a weak entropy of $A$, then

$$
\forall x \in E, \quad \psi_{0}(x) \leqslant \psi(x)<+\infty .
$$

Proposition 2.6 has the following partial converse.

THEOREM 2.7. Let $A$ be upper semicontinuous ${ }^{1}$ with compact values. Suppose $\forall x \in E, \psi_{0}(x)<+\infty$. Then $A$ is weakly dissipative and, in particular, $\psi_{0}$ is the smallest weak entropy of $A$.

Proof. For any $\varepsilon>0$, we define the function $\psi_{\varepsilon}: E \rightarrow R_{+}$by the formula

$$
\psi_{\varepsilon}(x)=\inf \left\{\sum_{n=0}^{\infty} d\left(x_{n}, x_{n+1}\right) \mid x_{0}=x \text { and } x_{n+1} \in B\left(A\left(x_{n}\right), \varepsilon\right)\right\}
$$

where $B(A(x), \varepsilon)=\{y \in E \mid d(y, A(x))<\varepsilon\}$.

We may also define $\psi: E \rightarrow R_{+}$by setting

$$
\psi(x)=\lim _{\varepsilon \rightarrow 0} \psi_{\varepsilon}(x) \text {. }
$$

Properties (2.8) and (2.9) are well defined for if $\varepsilon_{2}<\varepsilon_{1}$ then $\psi_{\varepsilon_{1}} \leqslant \psi_{\varepsilon_{2}} \leqslant \psi \leqslant \psi_{0}$.

Next, for each $\varepsilon>0$, select $x^{\varepsilon} \in B(A(x), \varepsilon)$ such that

$$
\psi_{\varepsilon}\left(x^{\varepsilon}\right)+d\left(x, x^{\varepsilon}\right) \leqslant \psi_{\varepsilon}(x)+\varepsilon .
$$

\footnotetext{
${ }^{1} \forall \varepsilon>0, \exists \delta>0$ such that $d\left(x^{\prime}, x\right)<\delta$ implies $A\left(x^{\prime}\right) \subset B(A(x), \varepsilon)$.
} 
Since $A(x)$ is compact, we may select a subsequence $\left\{x^{k}\right\} \in B(A(x), 1 / k)$ converging to some $x^{*} \in A(x)$. Also, since $A$ is upper semicontinuous, for any $\delta>0$ there exists $k_{0}$ with $\delta>1 / k_{0}$ and such that $\forall k \geqslant k_{0}, B\left(A\left(x^{k}\right), 1 / k\right) \subseteq$ $B\left(A\left(x^{*}\right), \delta\right)$. Thus, for $k \geqslant k_{0}$,

$$
\psi_{\delta}\left(x^{*}\right) \leqslant d\left(x^{*}, x^{k}\right)+\psi_{k}\left(x^{k}\right) .
$$

Combining (2.10) and (2.11) we have, for $k \geqslant k_{0}$,

$$
\psi_{\delta}\left(x^{*}\right)-d\left(x^{*}, x^{k}\right)+d\left(x, x^{k}\right) \leqslant \psi_{k}(x)+1 / k .
$$

Letting $k \rightarrow \infty$ in (2.8), we have

$$
\psi_{\delta}\left(x^{*}\right)+d\left(x, x^{*}\right) \leqslant \psi(x) .
$$

And finally, since $\delta$ is arbitrary,

$$
\psi\left(x^{*}\right)+d\left(x, x^{*}\right) \leqslant \psi(x) .
$$

Hence, $\psi$ is a weak entropy for $A$. Note that since $\psi \leqslant \psi_{0}$, Proposition 2.6 implies that $\psi=\psi_{0}$.

Theorems 2.4 and 2.7 imply the extension of the Banach-Picard fixed point theorem to multivalued maps.

Definition 2.15. We say that $A$ is Lipschitz with constant $\lambda>0$ if

$$
\forall x, y \in E, \quad A(y) \subseteq B(A(x), \lambda d(x, y)) .
$$

THEOREM 2.16. Let $A$ be Lipschitz with constant $1>\lambda>0$, and with compact images. Then it is weakly dissipative; hence it has a fixed point when $E$ is complete.

Proof. Since a Lipschitz multivalued map is upper semicontinuous, it suffices to check $\psi_{0}<+\infty$. Let $x \in E$. Since $A$ has compact images we may select a trajectory $\left\{x_{n}\right\}$ such that $d\left(x_{n}, x_{n+1}\right)=d\left(x_{n}, A\left(x_{n}\right)\right)$. Because $A$ is Lipschitz we have that

$$
\psi_{0}(x) \leqslant \sum_{n=0}^{\infty} d\left(x_{n}, x_{n+1}\right) \leqslant \sum_{n=0}^{\infty} \lambda^{n} d\left(x_{0}, x_{1}\right)<+\infty
$$

3. Example: weak entropies of $(1-S)^{-1}$. We give another example of a class of weakly dissipative dynamical systems that play an important role:

(3.1) let $E$ be a convex compact subset of a Banach space $U$, and

(3.2) let $S: E \rightarrow F(U)$ be an upper semicontinuous map with nonempty closed convex values.

The dynamical system we consider associates with any state $x_{n}$ solutions $x_{n+1}$ to

$$
x_{n+1}-x_{n} \in S\left(x_{n+1}\right) \text {. }
$$

This dynamical system is the "implicit finite difference scheme" of the continuous dynamical system $d x / d t \in S(x(t))$. If we set $A=(1-S)^{-1}: E \rightarrow \mathcal{T}(U)$, (3.3) may be written as $x_{n+1} \in A\left(x_{n}\right)$. We have to supplement conditions implying that $A$ maps $E$ to itself and that a given function $\varphi: E \rightarrow R_{+}$is a weak entropy.

We assume that

(3.4) $\varphi: E \rightarrow R_{+}$is convex and continuous on a neighborhood of $R$. 
So it has nonempty convex compact subdifferential $\partial \varphi(x) \subset U^{*}$ defined by

$$
\partial \varphi(x)=\left\{p \in U^{*} \mid \varphi(x)-\varphi(y) \leqslant\langle p, x-y\rangle, y \in U\right\}
$$

(see, for instance, R. T. Rockafellar [8] or J. P. Aubin [2, Chapter 4]). Let $B_{*}$ be the unit ball of $U^{*}$. We associate with $\varphi$ its "strong tangent cone" $R_{\varphi}(x) \subset U$ defined by

$$
\begin{aligned}
R_{\varphi}(x) & =\left(\partial \varphi(x)+B_{*}\right)^{-} \\
& =\left\{v \in U \text { such that }\langle p+q, v\rangle \leqslant 0, \forall p \in \partial \varphi(x), q \in B_{*}\right\} .
\end{aligned}
$$

We recall that the normal cone to $E$ at $x$ is defined by

$$
N_{E}(x)=\left\{p \in U^{*} \mid\langle p, x\rangle=\max \{\langle p, y\rangle \mid y \in E\}\right\}
$$

and that the tangent cone to $E$ at $x$ is defined by

$$
T_{E}(x)=N_{E}(x)^{-} \text {. }
$$

THEOREM 3.9. Let us assume that assumptions (3.1), (3.2) and (3.4) hold. If

$$
\forall x \in E, \quad S(x) \cap T_{E}(x) \cap R_{\varphi}(x) \neq \varnothing ;
$$

then $A=(1-S)^{-1}$ maps $E$ to itself and $\varphi$ is a weak entropy. Hence, for any $x_{0} \in E$, there exists a trajectory $\left\{x_{n}\right\}$ of (3.3) starting at $x_{0}$ and converging to a "critical point" $\bar{x} \in E$ of $S$ (i.e., satisfying $0 \in S(\bar{x})$ ).

Remark 3.11. A generalization of a Ky Fan theorem [6] by B. Cornet [4] shows that the condition $S(x) \cap T_{E}(x) \neq \varnothing$ implies that $S$ has critical points and that $A=(1-S)^{-1}$ maps $E$ to itself. (See J. P. Aubin [2, Chapter 15].)

We shall deduce the proof from the following Ky Fan inequality.

THEOREM 3.12(Ky FAN [6]). Let $E$ be a convex compact subset and let $f$ : $E \times E \rightarrow R$ satisfy

(i) $\forall x \in E, y \rightarrow f(x, y)$ is concave,

(ii) $\forall y \in E, x \rightarrow f(x, y)$ is lower semicontinuous,

(iii) $\forall y \in E, f(y, y) \leqslant 0$.

Then there exists $\bar{x} \in E$ satisfying $\sup \{f(\bar{x}, y) \mid y \in E\} \leqslant 0$ (for an elementary proof, see J. P. Aubin [1, Chapter 7, §5]).

Proof. For proving that $A$ maps $E$ to itself and that $\varphi$ is a weak entropy, we have to show that when $x_{0} \in E$ is given, there exists $\bar{x} \in E$ satisfying

$$
\bar{x}-x_{0} \in S(\bar{x}) \text { and }\left\|\bar{x}-x_{0}\right\| \leqslant \varphi\left(x_{0}\right)-\varphi(\bar{x}) .
$$

We denote by $\sigma(S(x), p)=\sup \{\langle p, v\rangle \mid v \in S(x)\}$ the support function of the closed convex set $S(x)$.

We note that the separation theorem implies that $x-x_{0} \in S(x)$ if and only if $\left\langle p, x-x_{0}\right\rangle \leqslant \sigma(S(x), p)$ for all $p \in U^{*}$. We also note that

$$
\left\|x-x_{0}\right\|-\varphi\left(x_{0}\right)+\varphi(x)=\sup _{\|q\|_{*}=1}\left(\left\langle q, x-x_{0}\right\rangle-\varphi\left(x_{0}\right)+\varphi(x)\right) .
$$

Let us assume that property (3.13) is false: i.e., that for all $x \in X$, either

$$
\left\|x-x_{0}\right\|-\varphi\left(x_{0}\right)+\varphi(x)>0 \text { or } x-y_{0} \notin S(x) \text {. }
$$


In other words, we can write

$$
E=\bigcup_{\|q\|_{*}=1} V_{q} \cup \bigcup_{p \in U^{*}} V_{p}
$$

where

$$
\begin{aligned}
& V_{q}=\left\{x \in X \mid\left\langle q, x-x_{0}\right\rangle-\varphi\left(x_{0}\right)+\varphi(x)>0\right\}, \\
& V_{p}=\left\{x \in X \mid\left\langle-p, x-x_{0}\right\rangle>\sigma(S(x),-p)\right\} .
\end{aligned}
$$

The subsets $V_{q}$ are open since $\varphi$ is lower semicontinuous. The upper semicontinuity of $S$ implies the upper semicontinuity of the functions $x \rightarrow \sigma(S(x), p)$; therefore, the subsets $V_{p}$ are open. Since $E$ is compact, it can be covered by $n$ open sets $V_{p_{i}}$ and $m$ open sets $V_{q_{j}}$. Let $\left\{\alpha_{i}, \beta_{j}\right\}, i=1, \ldots, n, j=1, \ldots, m$, be a continuous partition of unity subordinate to this covering. Let us define $f$ on $E \times E$ by

$$
\begin{aligned}
f(x, y)= & \sum_{j=1}^{n} \alpha_{j}(x)\left(\left\langle q_{j}, x-y\right\rangle+\varphi(x)-\varphi(y)\right) \\
& -\sum_{i=1}^{m} \beta_{i}(x)\left\langle p_{i}, x-y\right\rangle .
\end{aligned}
$$

It is clear that $f$ satisfies the assumptions of the Ky Fan theorem; therefore, there exists $\bar{x} \in E$ satisfying

$$
\forall y \in E, \quad f(\bar{x}, y) \leqslant 0 .
$$

Let us set

$$
\bar{q}=\sum_{j=1}^{n} \alpha_{j}(\bar{x}) q_{j}, \quad \bar{\lambda}=\sum_{j=1}^{n} \alpha_{j}(\bar{x}), \quad \bar{p}=\sum_{i=1}^{m} \beta_{i}(\bar{x}) p_{i}
$$

Inequality (3.15) amounts to saying that $\bar{x} \in E$ minimizes $\bar{\lambda}_{\varphi}(y)+\langle\bar{q}, y\rangle-$ $\langle\bar{p}, y\rangle$ on $E$. Since $\varphi$ is convex and continuous at a point of $E$ the standard duality theorem of convex analysis (see, for instance, J. P. Aubin [2, Chapter 14, Theorem 1.1], and R. T. Rockafellar [8]), implies that

$$
\bar{p} \in \bar{\lambda} \partial \varphi(\bar{x})+\bar{q}+N_{E}(\bar{x})
$$

Let us assume that $\alpha_{j}(\bar{x})=0$ for all $j$. Then there exists at least an index $i$ such that $\beta_{i}(\bar{x})>0$. For each index $i$ satisfying $\beta_{i}(\bar{x})>0$, we have $\bar{x} \in V_{p_{i}}$ and thus $\left\langle-p_{i}, \bar{x}-x_{0}\right\rangle>\sigma\left(S(\bar{x}),-p_{i}\right)$. We derive that $\left\langle-\bar{p}, \bar{x}-x_{0}\right\rangle>\sigma(S(\bar{x}),-\bar{p})$. Now, $\bar{\lambda}=0, \bar{q}=0$ and (3.16) implies that $\bar{p} \in N_{E}(\bar{x})$. Since there exists $\bar{v} \in S(\bar{x}) \cap$ $T_{E}(\bar{x})$, by assumption (3.10) we obtain

$$
f\left(\bar{x}, x_{0}\right)=-\left\langle\bar{p}, \bar{x}-x_{0}\right\rangle>\sigma(S(\bar{x}),-\bar{p}) \geqslant-\langle\bar{p}, \bar{v}\rangle \geqslant 0,
$$

i.e., a contradiction of (3.15).

Hence $\alpha_{j}(\bar{x})>0$ for at least one index $i$ and thus $\bar{\lambda}>0$. Since $\bar{x} \in V_{q_{j}}$ when $\alpha_{j}(\bar{x})>0$, we deduce that $\left\langle q_{j}, \bar{x}-x_{0}\right\rangle+\varphi(\bar{x})-\varphi\left(x_{0}\right)>0$. So

$$
\left\langle\bar{q}, \bar{x}-x_{0}\right\rangle+\bar{\lambda}\left(\varphi(\bar{x})-\varphi\left(x_{0}\right)\right)>0 .
$$


Now, since $\|\bar{q}\| \leqslant \bar{\lambda}$, we deduce from (3.16) that $\bar{p} \in \bar{\lambda}\left(\partial \varphi(\bar{x})+B_{*}+N_{E}(\bar{x})\right)$. By (3.10), there exists $\bar{v} \in S(\bar{x}) \cap T_{E}(\bar{x}) \cap(\partial \varphi(\bar{x})+B)^{-}$. Thus (3.17) implies that

$$
\left.f\left(\bar{x}, x_{0}\right)\right\rangle-\left\langle\bar{p}, \bar{x}-x_{0}\right\rangle \geqslant \sigma(S(\bar{x}),-\bar{p}) \geqslant\langle-\bar{p}, \bar{v}\rangle \geqslant 0,
$$

i.e., a contradiction of (3.15).

So, $A$ maps $E$ to itself and is weakly dissipative. Since $S$ is closed, $A$ is also closed. Then Theorem 2.4 implies that for any $x_{0} \in E$, there exists a trajectory $\left\{x_{n}\right\}$ of (3.3) that converges to a fixed point $\bar{x}$ of $A$, which is a critical point of $S$.

4. Dissipative systems. As the theory of weakly dissipative systems allows one to conclude that fixed points exist, so the theory of dissipative systems gives stationary points $(A(x)=\{x\})$. We briefly discuss some aspects of that theory.

Definition 4.1. Given a multivalued map $A: E \rightarrow F(E)$ a function $\varphi: E \rightarrow R_{+}$is called an entropy of $A$ if

$$
\forall x \in E, \quad \forall y \in A(x), \quad d(x, y) \leqslant \varphi(x)-\varphi(y) .
$$

A map $A$ is called dissipative if it has an entropy.

We are able to characterize dissipative maps in terms of the function $\varphi_{*}$ : $E \rightarrow[0, \infty]$ defined by setting

$$
\varphi_{*}(x)=\sup \left\{\sum_{n=0}^{\infty} d\left(x_{n}, x_{n+1}\right) \mid\left\{x_{n}\right\} \in \mathcal{T}(A, x)\right\} .
$$

In particular, we have:

THEOREM 4.4. $A$ multivalued map $A$ is dissipative iff $\forall x \in E, \varphi_{*}(x)<$ $+\infty$. In this case $\varphi_{*}$ is the smallest entropy of $A$.

Proof. The result quickly follows from the observation that we may rewrite 4.3 as

$$
\varphi_{*}(x)=\sup \left\{d(x, y)+\varphi_{*}(y) \mid y \in A(x)\right\} .
$$

In order to study the stationary points of a dissipative map, it is convenient to introduce certain associated maps. For $A: E \rightarrow F(E)$ we define $H_{A}: E \rightarrow F(E)$ by setting

$$
H_{A}(x)=\bigcup_{\left\{x_{n}\right\} \in \mathscr{T}(A, x)}\left\{x_{n}\right\}
$$

and for each $x \in E$ we define $L_{A}: \mathcal{T}(A, x) \rightarrow F(E)$ by setting

$$
L_{A}\left(\left\{x_{n}\right\}\right)=\bigcap_{n>0} \overline{H_{A}\left(x_{n}\right)} .
$$

$L_{A}\left(\left\{x_{n}\right\}\right)$ is called the limit set of the trajectory.

The relevant information about these maps is contained in the following proposition ( $A$ need not be dissipative). 
Proposition 4.8. $\forall x \in x$ we have

(a) $x \in H_{A}(x)$,

(b) $\forall y \in H_{A}(x), H_{A}(y) \subseteq H_{A}(x)$,

(c) $A\left(H_{A}(x)\right) \subseteq H_{A}(x)$,

(d) If either $\forall x, H_{A}(x)$ is closed, or $A$ is lower continuous, ${ }^{2}$ then $A\left(L_{A}\left(\left\{x_{n}\right\}\right)\right) \subseteq L_{A}\left(\left\{x_{n}\right\}\right)$.

Proof. (a), (b) and (c) follow at once from the definition of $H_{A}$; (d) is a simple continuity argument.

The next theorem is the main technical result of this section.

THEOREM 4.9. Let $A$ be dissipative with entropy $\varphi$. Then for any $x \in E$ there exists $\left\{x_{n}\right\} \in \mathcal{T}(A, x)$ converging to some $\bar{x} \in E$ and such that $L_{A}\left(\left\{x_{n}\right\}\right)=\{\bar{x}\}$.

Before proving 4.9, we list some applications, the first of which follows at once from 4.8(d) and 4.9.

THEOREM 4.10. Let $A$ be dissipative and let either $A$ be lower semicontinuous or $H_{A}$ be closed, then $A$ has a stationary point.

We also have the following generalization of Caristi's theorem (Caristi [3] or Siegel [9]).

TheOREM 4.11. Let $A$ be dissipative with lower semicontinuous entropy $\varphi$; then $A$ has a stationary point.

Proof. Let $\Phi: E \rightarrow F(E)$ be defined by setting

$$
\Phi(x)=\{y \mid d(x, y) \leqslant \varphi(x)-\varphi(y)\} .
$$

Note: (a) $\Phi=H_{\Phi}$ is closed, and (b) $\forall x, A(x) \subseteq \Phi(x)$. Therefore by 4.10 , $\Phi$ has a stationary point, hence so does $A \subseteq \Phi$.

Finally we have:

THEOREM 4.12. Let $f: E \times U \rightarrow E$ be such that:

(a) for each $u \in U, f_{u}: E \rightarrow E$ is continuous;

(b) there exists $\varphi: E \rightarrow R_{+}$such that $\forall x \in E, \forall u \in U$,

$$
d(x, f(x, u)) \leqslant \varphi(x)-\varphi(f(x, u)) ;
$$

then there exists $\bar{x} \in E$ such that $\forall u \in U, f(\bar{x}, u)=\bar{x}$.

Proof. Let $A: E \rightarrow F(E)$ be defined by setting $A(x)=\{f(x, u) \mid u \in U\} . \varphi$ is an entropy for $A$, and by hypothesis (a) $A$ is lower semicontinuous, hence by $4.10 A$ has a stationary point $\bar{x}$. Checking the definition of $A, \bar{x}$ satisfies the conclusion of the theorem.

Proof of 4.9. Firstly one checks that $H_{A}$ is dissipative, and $\varphi$ is an entropy for $H_{A}$. Next, let

$$
\rho(x)=\inf \left\{\varphi(y) \mid y \in H_{A}(x)\right\}
$$

${ }^{2} \forall y \in A(x), \forall \varepsilon>0, \exists \delta>0$ such that $d\left(x^{\prime}, x\right)>\delta$ implies $B(y, \varepsilon) \cap A\left(x^{\prime}\right) \neq \varnothing$. 
The diameter $\delta\left(H_{A}(x)\right)=\sup \left\{d(y, z) \mid y, z \in H_{A}(x)\right\}$ satisfies the inequality

$$
\forall x \in E, \quad \delta\left(H_{A}(x)\right) \leqslant 2[\varphi(x)-\rho(x)]
$$

since

$$
\begin{aligned}
\delta\left(H_{A}(x)\right) & \leqslant \sup _{y, z \in H_{A}(x)}\{d(y, x)+d(x, z)\} \\
& \leqslant 2 \sup _{y \in H_{A}(x)}\{d(y, x)\} \leqslant 2[\varphi(x)-\rho(x)] .
\end{aligned}
$$

Choose $\left\{x_{n}\right\} \in \mathcal{T}\left(H_{A}, x\right)$ such that $\varphi\left(x_{n+1}\right) \leqslant \rho\left(x_{n}\right)+2^{-n}$. By $4.8(\mathrm{~b}), \rho\left(x_{n}\right)<$ $\rho\left(x_{n+1}\right)$ thus by 4.14

$$
\delta\left(H_{A}\left(x_{n+1}\right)\right) \leqslant 2\left[\varphi\left(x_{n+1}\right)-\rho\left(x_{n+1}\right)\right] \leqslant 2^{1-n} .
$$

Thus, again by $4.8(\mathrm{~b}),\left\{x_{n}\right\}$ is Cauchy and

$$
L_{A}\left(\left\{x_{n}\right\}\right)=\bigcap \overline{H_{A}\left(x_{n}\right)}=\bar{x}=\lim _{\rightarrow} x_{n} .
$$

As a final application we can prove the following theorem of Ekeland [5].

TheOREM 4.15. Let $\varphi: E \rightarrow R_{+}$be lower semicontinuous. For any $\varepsilon>0$, let $y_{\varepsilon} \in E$ be such that $\varphi\left(y_{e}\right) \leqslant \inf _{y \in E} \varphi(y)+\varepsilon$. Then there exists $x_{\varepsilon} \in E$ such that

$$
d\left(x_{\varepsilon}, y_{\varepsilon}\right) \leqslant \sqrt{\varepsilon} \text { and } \varphi\left(x_{\varepsilon}\right)=\min _{x \in E}\left(\varphi(x)+\sqrt{\varepsilon} d\left(x_{\varepsilon}, x\right)\right) \text {. }
$$

ProOF. Let

$$
A(x)=H_{A}(x)=\{y \in E \mid d(x, y) \leqslant \varphi(x) / \sqrt{\varepsilon}-\varphi(y) / \sqrt{\varepsilon}\} .
$$

By 4.11, $A$ has a stationary point $x_{\varepsilon}$. The result follows from the definition of $A$.

\section{REFERENCES}

1. J. P. Aubin, Applied abstract analysis, Wiley Interscience, New York, 1977.

2. __ Mathematical methods of game and economic theory, North-Holland, Amsterdam, 1978.

3. J. Caristi, Fixed point theorems for mappings satisfying inwardness conditions, Trans. Amer. Math. Soc. 78 (1972), 186-197.

4. B. Cornet, Paris avec handicaps et théorèmes de surjectivité de correspondences, C.R. Acad. Sci. Paris Sér. A-B 281 (1975), 479-482.

5. I. Ekeland, On the variational principle, J. Math. Anal. Appl. 147 (1974), 324-353.

6. Ky Fan, A minimax inequality and applications, Inequalities. III, O. Shisha, Ed., Academic Press, New York, 1972, pp. 103-113.

7. E. Polac, Computational methods in optimization, Academic Press, New York, 1974.

8. R. T. Rockafellar, Conjugate duality and optimization, SIAM, Philadelphia, Pa., 1974.

9. J. Siegel, A new proof of Caristi's fixed point theorem, Proc. Amer. Math. Soc. 66 (1977), 54-56.

10. J. C. Willems, Dissipative dynamical systems, Arch. Rational Mech. Anal. 45 (1972), 321-351.

11. W. Zangwill, Nonlinear programming, Prentice-Hall, Englewood Cliffs, N.J., 1969.

Département de Mathématique, Université de Paris, Dauphine, France

Department of Mathematics, University of Missouri, St. Louis, Missouri 63121 